\title{
Articular cartilage regeneration with autologous marrow aspirate and hyaluronic acid: an experimental study in a goat model
}

\begin{abstract}
Purpose:

The purpose of the study was to determine whether postoperative intra-articular injections of autologous marrow aspirate (MA) and hyaluronic acid (HA) after subchondral drilling resulted in better cartilage repair as assessed histologically by Gill scoring.

Methods:

In a goat model we created a 4-mm full-thickness articular cartilage defect in the stifle joint (equivalent to $1.6 \mathrm{~cm}$ in the human knee) and conducted subchondral drilling. The animals were divided into 3 groups: group A (control), no injections; group B (HA), weekly injection of $1 \mathrm{~mL}$ of sodium hyaluronate for 3 weeks; and group $\mathrm{C}$ (HA + MA), similar to group B but with $2 \mathrm{~mL}$ of autologous MA in addition to HA. MA was obtained by bone marrow aspiration, centrifuged, and divided into aliquots for cryopreservation. Fifteen animals were equally divided between the groups and sacrificed 24 weeks after surgery, when the joint was harvested, examined macroscopically and histologically.
\end{abstract}

Results:

Of the 15 animals, 2 from group A had died of non-surgery-related complications and 1 from group $\mathrm{C}$ was excluded because of a joint infection. In group A the repair constituted mainly scar tissue, whereas in group B there was less scar tissue, with small amounts of proteoglycan and type II collagen at the osteochondral junction. In contrast, repair cartilage from group C animals showed almost complete coverage of the defect with evidence of hyaline cartilage regeneration. Histology assessed by Gill scoring was significantly better in group $\mathrm{C}$ with 1way analysis of variance yielding an F statistic of 10.611 with a $P$ value of .004 , which was highly significant.

Conclusions:

Postoperative intra-articular injections of autologous MA in combination with HA after subchondral drilling resulted in better cartilage repair as assessed histologically by Gill scoring in a goat model.

Clinical Relevance:

After arthroscopic subchondral drilling, this novel technique may result in better articular cartilage regeneration.

Keyword: Arthroscopic subchondral drilling; Articular cartilage; Hyaline cartilage; Hyaluronic acid; Mesenchymal stem cells; Microfracture 